\title{
CNS LESIONS IN VON HIPPEL-LINDAU SYNDROME
}

Ninety-seven gene carriers of von Hippel-Lindau syndrome (HLS) were studied at the University of Freiburg, Germany, to evaluate the prevalence and location of CNS lesions. Hemangioblastomas of the CNS were found in 43 patients $(44 \%)$. Of a total of 93 hemangioblastomas found in 43 patients (44\%), $74 \%$ were intracranial and $26 \%$ were in the spinal cord. Seventy-five percent were cystic and $25 \%$ solid. Multiple lesions occurred in $42 \%$ of HLS hemangioblastomas but in none of 51 CNS hemangioblastomas without HLS. Cerebella hemangioblastomas were the major cause of death in $82 \%$ of the patients with HLS (Neumann HPH et al. Central nervous system lesions in von Hippel-Lindau syndrome. J Neurol Neurosurg Psychiatry Oct 1992; 55:898901). (Correspondence: Dr. Neumann, Medizinische Klinik, Hugstetter Str 55, D-7800 Freiburg, Germany.)

COMMENT. Other manifestations of von Hippel-Lindau syndrome were as follows: retinal angiomatosis - $44 \%$, renal cysts - $47 \%$, pheochromocytoma - 5\%, pancreatic cysts - $30 \%$, and epididymal cystadenoma - $15 \%$. The MRI and gadolinium-enhanced MRI have improved the detection of small CNS lesions which are the initial manifestations of the syndrome and the largest percentage of patients. Multifocal tumor development and recurrence are serious problems in management and prognosis.

\section{METABOLIC DISORDERS}

\section{PROPIONIC ACIDEMIA: PROGNOSIS}

The neurologic outcome of 20 patients with propionic acidemia was evaluated at the Medical Unit, Institute of Child Health, London, England. In 11 patients who presented in the first week of life, the death rate was high and all were mentally retarded (IQ less than 60), and 3 had mild chorea or dystonia. Of 9 patients with onset after the neonatal period, 4 had a severe movement disorder that evolved following an episode of metabolic derangement. In the late onset group, CT disclosed transient basal ganglia lucencies after episodes of metabolic decompensation. CSF neurotransmitter metabolites were unchanged (Surtees RAH et al. Neurologic outcomes of propionic acidemia. Pediatr Neurol Sept/Oct 1992; $\underline{8}: 333-337)$. (Communications: Dr. Surtees, Medical Unit, Institute of Child Health, 30 Guilford Street, London WC1N 1EH, England.)

COMMENT. A biotin-responsive propionic acidemia in a newborn is reported from Buenos Aires, Argentina and Paris, France (Cayssials AE et al. Pediatr Neurol Sept/Oct 1992; $: 409$ (abstract)). The infant presented at the 4 th day of life with partial feeding rejection, drowsiness, tachypnea, hypothermia and hepatomegaly. He developed generalized tonic-clonic seizures and required mechanical respiratory support. Enzyme studies in fibroblasts showed a profound deficiency of propionyl-CoA carboxylase activity and normal values for pyruvate carboxylase. Lab results showed severe metabolic acidosis and 
ketonuria. Treatment with carnitine and biotin was followed by clinical improvement and normal urine organic acids. One patient in the early onset group of the London study was treated with biotin and lived to 6 years of age. The outcome for patients with propionic acidemia is generally poor, although there is a wide variation.

Propionic acidemia (ketotic hyperglycinemia) should be distinguished from non-ketotic hyperglycinemia which is characterized by hypotonia, lethargy and seizures beginning on the first day of life. Some infants succumb within a few weeks, whereas others develop mental retardation and extrapyramidal signs (Menkes JH. Textbook of Child Neurology 3rd Ed., Philadelphia, Lea \& Febiger 1981).

\section{LEIGH ENCEPHALOPATHY}

Histologic and biochemical analyses of muscle biopsies from 33 patients with Leigh encephalopathy were performed at the National Institute of Neuroscience, Tokyo and Tokushima University School of Medicine, Japan. Cytochrome c oxidase activity was decreased or absent in 7 patients (21\%), 10 patients $(30 \%)$ had biochemical defects including 2 with pyruvate dehydrogenase complex, 4 with cytochrome c oxidase, 1 with NADHcytochrome c reductase and 3 with multiple complex deficiencies. None had DNA deletions in the muscle mitochondria. (Nagai $\mathrm{T}$ et al. Leigh encephalopathy: histologic and biochemical analyses of muscle biopsies. Pediatr Neurol Sept/Oct 1992; $\underline{8}: 328-332$.) (Communications: Dr. Nagai, Division of UItrastructural Research, National Institute of Neuroscience, NCNP, Kodaira, Tokyo 187, Japan.)

COMMENT. A mitochondrial DNA mutation in the ATPase 6 gene was reported in 7 of 40 patients with neuropathologically or MRI defined Leigh syndrome but no known biochemical defect (Santorelli FM et al. Ann Neurol Sept $1992 ; \underline{32}: 467)$. A high abundance of the "NARP" mutation (neuropathy, ataxia, and retinitis pigmentosa) can cause Leigh syndrome and should be looked for in patients without biochemical defects. In another report, a 7 year old girl presented with a partial pyruvate carboxylase deficiency and basal ganglia lesions compatible with Leigh's disease (Schrank WI et al. Ann Neurol Sept $1992 ; 32: 468)$. The biochemical defects in Leigh encephalopathy are probably heterogeneous.

\section{NEUROPATHIES}

\section{HMSN I: EARLY DIAGNOSIS}

The value of nerve conduction velocities in the detection of hereditary motor sensory neuropathy type I (HMSN I) in children at risk was determined in 36 children under 10 years of age at the University of Western Ontario, London, Ontario, Canada. Clinical signs and slowed motor nerve conduction velocities were found in 17 of the 36 children who had 1 parent with 